(C) [2009] IEEE. Reprinted, with permission, from Mohd Ramli Huda Adibah., Sandrasegaran, Kumbesan., Basukala, Riyaj \& Wu Leijia 2009, 'Modeling and simulation of packet scheduling in the downlink long term evolution system', Proceedings of 15th Asia-Pacific Conference on Communications, APCC 2009, pp. 68-71. This material is posted here with permission of the IEEE. Such permission of the IEEE does not in any way imply IEEE endorsement of any of the University of Technology, Sydney's products or services. Internal or personal use of this material is permitted. However, permission to reprint/republish this material for advertising or promotional purposes or for creating new collective works for resale or redistribution must be obtained from the IEEE by writing to pubs-permissions@ieee.org. By choosing to view this document, you agree to all provisions of the copyright laws protecting it. 


\title{
Modeling and Simulation of Packet Scheduling in the Downlink Long Term Evolution System
}

\author{
Huda Adibah Mohd Ramli, Kumbesan Sandrasegaran, Member, IEEE, Riyaj Basukala, Leijia Wu \\ Faculty of Engineering and Information Technology, \\ University of Technology, Sydney \\ [HudaAdibah.MohdRamli, kumbes, lewu]@eng.uts.edu.au \\ Riyaj.Basukala@uts.edu.au
}

\begin{abstract}
Long term evolution (LTE) is the next generation wireless system that uses orthogonal frequency division multiple access (OFDMA) technology in the downlink. Packet scheduling becomes paramount as the wireless systems are moving towards all IP transmissions. Since LTE system is relatively new, very little work has been published to date that can precisely model and simulate the downlink LTE system environment. This paper provides detailed descriptions on modeling and simulation of packet scheduling in the downlink LTE system using a $\mathrm{C}++$ simulation tool. The tool accurately modeled the wireless system parameters and can be used for study of optimization of the LTE system.
\end{abstract}

Index Terms - LTE, packet scheduling, modeling and simulation, OFDMA.

\section{INTRODUCTION}

LTE is a new radio access technology proposed by the third generation partnership project (3GPP) to provide a smooth migration towards fourth generation (4G) wireless systems. LTE is envisaged to provide better quality of communications by providing higher data rates, reduced latency and increased in capacity and coverage. In the downlink, LTE uses OFDMA technology such that each user is allocated a portion of available bandwidth (a group of sub-carriers) based on its requirements, current load and system configuration. LTE network architecture consists of only eNodeBs that perform all radio related functions. The mechanisms that are responsible for managing and controlling radio resources are known as radio resource management (RRM). Packet scheduling is one of the RRM mechanisms and it is responsible for intelligent selection of a user's packets to use the appropriately selected radio resources.

Computer simulation is a well established technique that makes modeling and investigation of a large scale wireless system feasible [1]. Though there are many simulation tools developed to evaluate the performance of the wireless systems, the tools are too general and not suitable for simulating the downlink LTE environment. Moreover, packet scheduling in this system is a relatively new area of research and very little work has been published to date which are related to modeling and simulation of packet scheduling for the stated system. This paper provides detailed descriptions on the modeling and simulation of packet scheduling in the downlink LTE system using a $\mathrm{C}++$ simulation tool.
The remainder of this paper is organized as follows. Section II describes the downlink LTE system model followed by discussions on packet scheduling algorithms in Section III. The packet scheduling simulation model is discussed in Section IV while Section V contains the environments and results of the simulation. Section VI concludes the paper.

\section{Downlink LTe System Model}

The resource that is allocated to a user in the downlink LTE system is defined in both frequency and time domain and is called a resource block (RB). In the frequency domain, the $\mathrm{RB}$ consists of 12 consecutive sub-carriers $(180 \mathrm{kHz}$ total bandwidth) and in the time domain it is made up of one time slot of $0.5 \mathrm{~ms}$ duration. A time slot consists of 7 OFDM symbols. Packet scheduling is performed at eNodeB at $1 \mathrm{~ms}$ interval (transmit time interval, TTI) and two consecutive RBs (in time domain) are assigned to a user.

In this paper, it is assumed that users report their channel conditions (signal-to-noise-ratio, SNR) on each RB to serving eNodeB at each TTI and channel reporting is assumed to be error-free. The reported channel conditions are determined based on the computed SNR values of the sub-carrier located at the centre frequency of each RB. All sub-carriers within a $\mathrm{RB}$ are assumed to have the same SNR values as the subcarrier located at the centre frequency. Based on the received SNR value, the serving eNodeB maps the achievable data rate (which is the number of bits in two consecutive RBs) that a user can support at each TTI.

It is assumed in this paper that each user is assigned a buffer of infinite capacity at the eNodeB. Packets that arrive into the buffer are time stamped and queued for transmission based on a first-in-first-out (FIFO) basis. At each TTI and on each RB, packet scheduler (located at the eNodeB) selects a user with the highest priority based on packet scheduling algorithm and transmits packets to the user based on the reported SNR value discussed earlier. Fig. 1 shows a generalized model of packet scheduling algorithm in the downlink LTE system. 


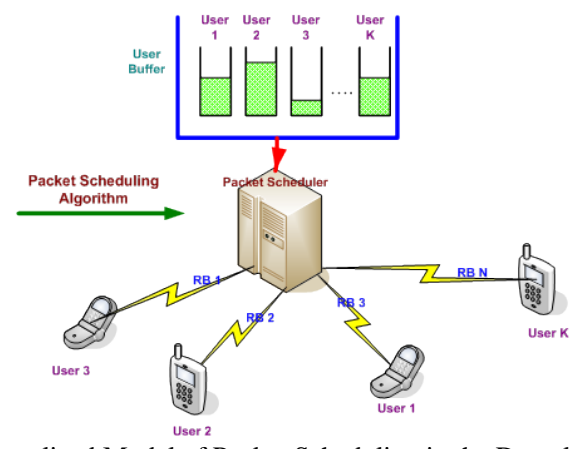

Fig. 1: Generalized Model of Packet Scheduling in the Downlink 3GPP LTE System

III. Packet Scheduling Algorithms

Packet scheduling algorithm represents an approach on how to transmit packet efficiently by using appropriate scheduling criteria such that specified performance metrics are satisfied. In a non-real time (NRT) service environment, channel condition is the most common scheduling criterion being considered while system throughput and fairness are being used when evaluating the performance of packet scheduling algorithms. In this paper, system throughput and fairness are defined as below:

System throughput is defined as the total size of transmitted packets per second and can be mathematically expressed as:

systemthroughput $=\frac{1}{T} \sum_{i=1}^{K} \sum_{t=1}^{T}$ ptransmit $_{i}(t)$

where $\operatorname{ptransmit}_{i}(t)$ is the size of transmitted packets of user $i$ at time $t, K$ is the total number of users and $T$ is the total simulation time.

Fairness is defined as the difference in service levels (total size of transmitted packets) between the most and the least served users for a given algorithm over a given time frame [2]. The algorithm with the highest fairness value obtained from the following equation indicates that it has the best fairness performance over other algorithms.

fairness $=1-\frac{\text { ptotaltransmit }_{\max }-\text { ptotaltransmit }_{\min }}{\sum_{i=1}^{K} \sum_{t=1}^{T} p s i z e_{i}(t)}$

where ptotaltransmit $t_{\max }$ and ptotaltransmit $t_{\min }$ are the total size of the transmitted packets of the most and the least served users respectively for each evaluated algorithm while $p s i z e_{i}(t)$ is the size of all packets of user $i$ that arrived at eNodeB buffer at time $t$.

The maximum rate (Max-Rate) [3], proportional fair (PF) [4] and round robin (RR) [3] are the packet scheduling algorithms developed to support for NRT services in single carrier wireless systems. The Max-Rate algorithm always selects the user with the highest reported SNR value. This algorithm efficiently utilizes radio resource because it selects packets of users with a good channel conditions for transmission. On the other hand, the algorithm has low fairness performance as it deprives a user with low SNR value from receiving any packets unless the user's channel condition improved.

Given that fairness has been an issue in Max-Rate algorithm, the RR algorithm was developed to address the problem. RR meets fairness by allocating an equal share of packet transmission time to each user. However, throughput performance degrades significantly as the algorithm does not rely on the reported SNR values when transmitting the selected user's packets.

To provide a balance between throughput and fairness, PF algorithm was proposed. PF was originally developed to support the NRT service in a code division multiple access high data rate (CDMA-HDR) system. The metric $k$ is defined as the ratio of:

$k=\arg \max \frac{r_{i}(t)}{R_{i}(t)}$

and;

$R_{i}(t)=\left(1-\frac{1}{t_{c}}\right) * R_{i}(t-1)+\frac{1}{t_{c}} * r_{i}(t-1)$

where $r_{i}(t)$ and $R_{i}(t)$ are the achievable data rate and the average data rate of user $i$ at time $t$, respectively, and $t_{c}$ is the size of an update window. $r_{i}(t-1)=0$ if user $i$ is not selected for transmission at time $t-1$. The size of the update window enable PF algorithm to maximize throughput and satisfy fairness for each user.

\section{Packet Scheduling - Simulation Model}

A C++ simulation tool was developed to model and simulate packet scheduling in the downlink LTE system. The tool consists of five modules and describe as follows:

\section{A. Mobility Module}

This module simulates the mobility of users within a single hexagonal cell. Users are initially distributed randomly within the simulation area and are moving at speeds between 1-100 $\mathrm{km} / \mathrm{h}$ in a random direction. The new location of user $i$ $\left(\operatorname{loc}_{i}(t+1)\right)$ is determined using the following equation:

$\operatorname{loc}_{i}(t+1)=\operatorname{loc}_{i}(t)+\left(v_{i}(t)^{*} d \operatorname{dir}_{i}(t)\right)$

where $v_{i}(t), d i r_{i}(t)$ are the speed and direction of user $i$ at time $t$. A constant speed and direction are assigned to a user but varied among users. A wrap-around method is employed at the cell boundary to ensure users always remain within the simulated area.

\section{B. Radio Propagations Module}

This module uses the speed and location of a user (generated by the mobility module) in order to compute pathloss, shadow fading and multi-path fading. Hata model [5] is used to compute pathloss for each user and consists of the following equations:

$$
\begin{aligned}
& p l_{i}(t)=46.3+33.9 * \log _{10}(f)-13.82 * \log _{10}\left(h_{b}\right) \\
& -a\left(h_{m}\right)+\left(44.9-6.55 * \log _{10}\left(h_{b}\right)\right) * \log _{10}\left(d_{i}(t)\right)
\end{aligned}
$$


and:

$$
a\left(h_{m}\right)=\left(1.1 * \log _{10}(f)-0.7\right) * h_{m}-\left(1.56 * \log _{10}(f)-0.8\right)
$$

where $p l_{i}(t)$ and $d_{i}(t)$ are the path loss (in $\mathrm{dB}$ ) and distance (in $\mathrm{km}$ ) of user $i$ at time $t$, respectively. $f$ is the frequency of the transmission (in MHz), $h_{b}$ and $h_{m}$ are the heights of eNodeB and user terminal (in meters), respectively and $a\left(h_{m}\right)$ is the mobile antenna correction factor.

The approach proposed by Gudmundson [6] is used to compute shadow fading gain in which it is modelled as a correlated log-normal distribution with 0 mean and $8 \mathrm{~dB}$ standard deviation. The equation used to determine shadow fading gain for user $i$ at time $t+1\left(\xi_{\mathrm{i}}(t+1)\right)$ is as follows:

$$
\xi_{i}(t+1)=\rho_{i}(t) \cdot * \xi_{i}(t)+\sigma *\left(\sqrt{1-\rho_{i}(t)^{2}} \cdot\right) * W(t)
$$

where $\sigma$ is the shadow fading standard deviation, and $W(t)$ is a Gaussian random variable at time $t$. The shadow fading autocorrelation function of user $i$ at time $t\left(\rho_{i}(t)\right)$ is computed using the equation below:

$\rho_{i}(t)=\exp \left(\frac{-v_{i}(t)}{d_{0}}\right)$

where $v_{i}(t)$ is the speed of user $i$ at time $t$, and $d_{0}$ is the shadow fading correlation distance.

A C++ program developed by Komninakis [7] which follows Rayleigh distribution is used to generate multi-path fading gains. It is assumed in this paper that, at any time instant multi-path fading gains vary on each $\mathrm{RB}$ whereas pathloss and shadow fading gains are fixed on each RB.

\section{SNR to Data Rate Mapping Module}

This module computes SNR value and maps it to the associated achievable data rate. The pathloss, shadow fading and multi-path fading gains (as discussed earlier) are used to determine channel gains and hence SNR values of each user and on each RB. The channel gain of user $i$ on RB $j$ at time $t$ $\left(\operatorname{Gain}_{i, j}(t)\right)$ is computed using the following equation:

$\left.\operatorname{Gain}_{i, j}(t)=10^{\left(\frac{p_{i}(t)}{10}\right)} * 10^{\left(\frac{\xi_{i}(t)}{10}\right)} * 10^{\left(\frac{m_{p a t h}, j}{10}(t)\right.}\right)$

where mpath $_{i, j}(t)$ is the multi-path fading gain of user $i$ on a $\mathrm{RB} j$ at time $t$. From the computed channel gain, the SNR value $\left(\gamma_{i, j}(t)\right)$ of user $i$ on RB $j$ at time $t$ is computed using the approach proposed in [8] and is given as below:

$\gamma_{i, j}(t)=\frac{P_{\text {total }} * \operatorname{Gain}_{i, j}(t)}{N_{R B}\left(I+N_{o}\right)}$

where $P_{\text {total }}$ is the total eNodeB downlink power, $N_{R B}$ is the number of RBs, $N_{o}$ is the thermal noise and $I$ is the inter-cell interference. The inter-cell interference is dependent on user's location and since the simulation time is very small, the user's location does not vary much and so the inter-cell interference is assumed constant.

The approach proposed in [9] is used to compute the number of bits per symbol of user $i$ at time $t$ on a sub-carrier

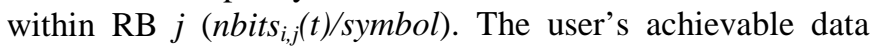
rate $\left(d r_{i}(t)\right)$ at time $t$ on two consecutive RBs are determined using: $d r_{i}(t)=\frac{\text { nbits }_{i, j}(t)}{\text { symbol }} * \frac{\text { nsymbols }}{\text { slot }} * \frac{\text { nslots }}{T T I} * \frac{n s c}{R B}$

where nsymbols/slot is the number of symbols per slot, $n s l o t / T T I$ is the number of slots per TTI and $n s c / R B$ is the number of sub-carriers per RB. The minimum SNR value and the associated achievable data rate used in this module are given in Table I.

\section{Traffic Generator Module}

This module generates the NRT packets for each user and queues the packets in the buffer. Web browsing is used to model NRT service and the parameters used in this module are based on 3GPP specifications [10].

\section{E. Packet Scheduler Module}

At each TTI and on each RB, this module selects a user with the highest priority based on a packet scheduling algorithm. The packets of the selected user (generated by traffic generator module) are taken from the buffer and transmitted over the assigned RB using the data rate given in Table I. Fig. 2 shows the interaction of each module within the simulation tool.

\section{Simulation Environment \& Results}

In this paper, a cell of $5 \mathrm{MHz}$ bandwidth and $2 \mathrm{GHz}$ carrier frequency are modeled. The cell is assumed to consist of 25 RBs and $K$ users and the users are simulated within $300 \mathrm{~m}$ radius. The serving eNodeB has a fixed location at the centre of the cell and controls all the available RBs. These RBs are to be shared by all users within the cell. At each TTI, two consecutive RBs can be assigned to a user. It is assumed that all sub-carriers are used for data transmission and equal transmit power is allocated on each RB. The downlink LTE system parameters used in this simulation are given in Table II while other simulation parameters are as described in Section II and IV.

The assumptions made in this simulation include:

- Each user has only one packet call throughout the simulation.

- A constant data rate of $168 \mathrm{kbps}$ is used in the RR algorithm.

TABLE I:

SNR TO DATA RATE MAPPING TABLE

\begin{tabular}{|ccc|}
\hline $\begin{array}{c}\text { Minimum SNR } \\
\text { Level (dB) }\end{array}$ & $\begin{array}{c}\text { Modulation and } \\
\text { Coding }\end{array}$ & $\begin{array}{c}\text { Data Rate } \\
\text { (kbps) }\end{array}$ \\
\hline 1.7 & QPSK (1/2) & 168 \\
\hline 3.7 & QPSK (2/3) & 224 \\
\hline 4.5 & QPSK (3/4) & 252 \\
\hline 7.2 & 16 QAM (1/2) & 336 \\
\hline 9.5 & 16 QAM (2/3) & 448 \\
\hline 10.7 & 16 QAM (3/4) & 504 \\
\hline 14.8 & 64 QAM (2/3) & 672 \\
\hline 16.1 & 64 QAM (3/4) & 756 \\
\hline
\end{tabular}




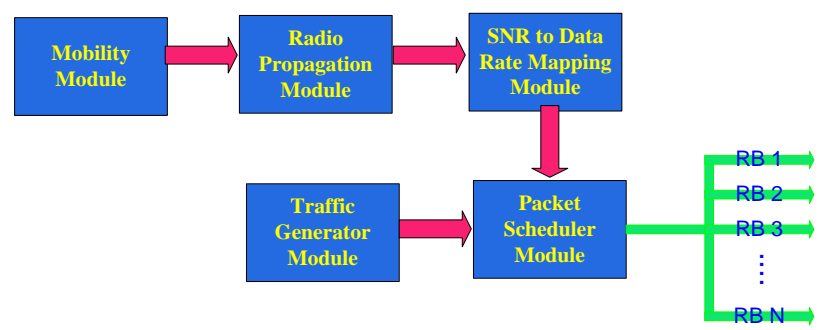

Fig. 2: Simulation Tool Block Diagram

A performance comparison of Max-Rate, RR and PF algorithms from NRT perspective was conducted using the simulation tool. Fig. 3 and Fig. 4 show system throughput and fairness performance of the three algorithms.

When compared with PF and RR, it can be seen in both figures that Max-Rate has the best system throughput but worst in fairness performance. Max-Rate utilizes the radio resources by always transmitting packets of a user with good channel conditions and starves the users with poor channel conditions from receiving their packets. Meanwhile, RR has the worst throughput performance for not considering channel conditions when transmitting users' packets but the best in fairness as it allocates equal share of resources to each user. PF performance falls in between the two extremes.

Based on the results, it can be concluded that, the tool is able to simulate packet scheduling in the downlink LTE system and provides the system throughput and fairness performance of Max-Rate, PF and RR algorithms similar to as discussed in Section III.

\section{Conclusions}

This paper models and simulate packet scheduling in the downlink LTE system using a $\mathrm{C}++$ simulation tool. The tool models the mobility and radio environment that are necessary when modeling the channel gains in the wireless system. Furthermore, the tool takes into account the SNR computations, SNR to data rate mapping as well as traffic generation such that packet scheduling can be performed efficiently using the tool. In the future, this tool will be used to evaluate the performance of packet scheduling algorithms developed for the downlink LTE system.

TABLE II:

LTE DOWNLINK SYSTEM PARAMETERS

\begin{tabular}{|cc|}
\hline Parameters & Values \\
\hline Carrier Frequency & $2 \mathrm{GHz}$ \\
\hline Bandwidth & $5 \mathrm{MHz}$ \\
\hline Number of Sub-carriers & 300 \\
\hline Number of RBs & 25 \\
\hline Number of Sub-carriers per RB & 12 \\
\hline Sub-Carrier Spacing & $15 \mathrm{kHz}$ \\
\hline Slot Duration & $0.5 \mathrm{~ms}$ \\
\hline Scheduling Time (TTI) & $1 \mathrm{~ms}$ \\
\hline Number of OFDM Symbols per Slot & 7 \\
\hline
\end{tabular}

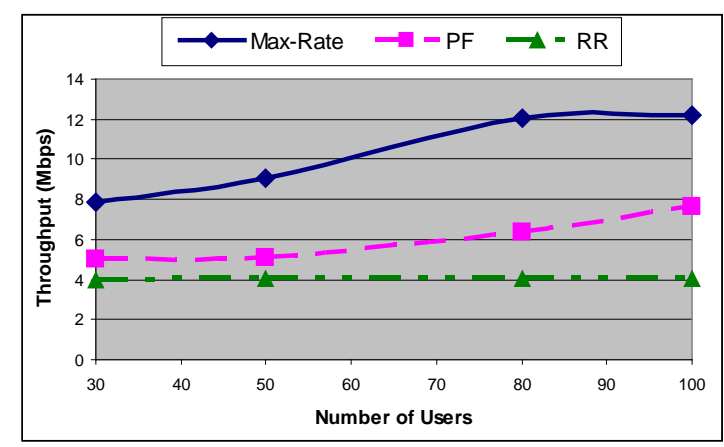

Fig. 3: Throughput vs. Number of Users

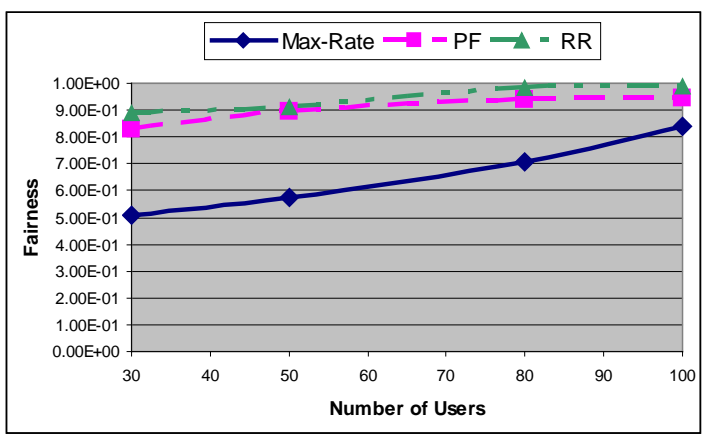

Fig. 4: Fairness vs. Number of Users

\section{REFERENCES}

[1] L. Bononi, M. Bracuto, G. D’Angelo, and L. Donatiello, "Performance Analysis of a Parallel and Distributed Simulation Framework for Large Scale Wireless Systems," ACM, 2004.

[2] A. Gyasi-Agyei and S.-L. Kim, "Comparison of Opportunistic Scheduling Policies in Time-Slotted AMC Wireless Networks," in 1st International Symposium on Wireless Pervasive Computing, 2006.

[3] E. Dahlman, S. Parkvall, J. Skold, and P. Beming, 3G Evolution: HSPA and LTE for Mobile Broadband, First ed.: Elsevier Ltd., 2007.

[4] A. Jalali, R. Padovani, and R. Pankaj, "Data Throughput of CDMAHDR a High Efficiency-High Data Rate Personal Communication Wireless System," in IEEE 51st Vehicular Technology Conference Proceedings, Tokyo, 2000, pp. 1854-1858.

[5] H. Holma and A. Toskala, WCDMA for UMTS: HSPA Evolution and LTE, Fourth ed.: John Wiley \& Sons Ltd., 2007.

[6] M. Gudmundson, "Correlation Model for Shadow Fading in Mobile Radio Systems," in Electronics Letters. vol. 27, 1991, pp. 2145-2146.

[7] C. Komninakis, "A Fast and Accurate Rayleigh Fading Simulator," in IEEE Globecom San Francisco, CA, 2003.

[8] K. Kim, G.-M. Yeo, B.-H. Ryu, and K. Chang, "Interference Analysis and Subchannel Allocation Schemes in Tri-Sectored OFDMA Systems," in IEEE 66th Vehicular Technology Conference, 2007, pp. 1857-1861.

[9] X. Qiu and K. Chawla, "On the Performance of Adaptive Modulation in Cellular Systems," in IEEE Transactions on Communications. vol. 47, 1999, pp. 884-895.

[10] 3GPP2, "cdma2000 Evaluation Methodology," December 10, 2004. 